Philippe Canguilhem

\title{
Zur Rekonstruktion improvisierter Mehrstimmigkeit der Renaissance am Fall der si placet-Praxis*
}

Nachdem Juan Martin de Riscos das Amt des maestro di capilla an der Kathedrale von Jaén in Andalusien von 1598 an bis zu seinem Tod bekleidet hatte, war die dortige Stelle im Jahr 1637 zunächst vakant. Um das Kapitel im Zuge der anstehenden Neubesetzung zu unterstützen, bat man den erst kurz zuvor als Organist der Kathedrale eingestellten, in Spanien weithin bekannten Francisco Correa de Arrauxo darum, eine Abhandlung über die Art des Verfahrens zu verfassen, das die Bewerber durchlaufen sollten. In dem daraufhin entstandenen Dokument beschreibt Correa zunächst die zu jener Zeit gängige Vorgehensweise an spanischen Kathedralen im Detail und ergänzt diese um eigene Vorschläge zur Modifizierung des Verfahrens. Es ist daher ein eindrückliches Zeugnis für die Ansprüche, die gegen Ende der Renaissance an die Kapellmeister von Kathedralen gestellt wurden. ${ }^{1}$

Correa beginnt mit der Beschreibung dreier Fertigkeiten, deren Beherrschung für einen guten Kapellmeister unabdingbar war und daher unter Beweis gestellt werden sollte. Sie umfassen

1. das Extempore-Singen des Kontrapunkts (Echar contrapunto de repente)

2. das Improvisieren einer dritten, vierten und fünften Stimme (Echar terceras, quartas y quintas vozes)

3. Expertise im Bereich der Musiktheorie (Estar bien en las téoricas de todas las materias musicales).

Das Beherrschen dieser Fähigkeiten war nach Correas Auffassung notwendig für die Erfüllung der drei Hauptaufgaben des Kapellmeisters, die darin bestanden

1. das Musizieren am Pult anzuleiten (Regir bien el fascitol)

2. in lateinischer wie auch in spanischer Sprache zu komponieren (Componer latín y romance)

3. zu unterrichten (Enseñar $)^{2}$

* Übersetzung von Chantal Köppl (Universität Mainz).

1 Correas Parecer wurde in Dionisio Preciado, »Un documento nuevo del gran organista andaluz Francisco Correa de Araújo (1584-1654)«, in: Codice 5 (1990), S. 43-48 veröffentlicht. Preciado datiert in seinem Beitrag ferner den Tod Correas auf Januar 1637 und korrigiert damit das fälschliche Todesjahr 1643, das sich in der älteren Forschungsliteratur gelegentlich findet. $\mathrm{Zu}$ Correas Werdegang in Jaén vgl. Robert Stevenson, »Francisco Correa de Arauxo. New light on his career«, in: Revista Musical Chilena 103 (1968), S. 9-42.

2 Preciado, »Un documento nuevo« (wie Anm. 1), S. 46. 
Der einleitende Teil seines Berichts wirft eine Reihe von Fragen auf. Das aus heutiger Perspektive offensichtlichste Problem stellt die Tatsache dar, dass vom maestro de capilla zwar erwartet wurde, in lateinischer wie in spanischer Sprache zu komponieren, die Befähigung dazu jedoch offensichtlich anhand einer Reihe von Kontrapunktübungen, in Form von Improvisationen, festgestellt werden sollte.

»Echar contrapunto de repente« - das wörtlich übersetzt «den Kontapunkt ex tempore hinwerfen» bedeutet - war vom 15. bis in das 18. Jahrhundert hinein weit verbreitete Praxis und in ganz Europa tagtägliche Realität für die Sänger, die den Chören von Kathedralen, Kollegiatskirchen oder den Kapellen von Fürstenhäusern angehörten. In Spanien unter dem Begriff «contrapunto» bekannt, unterschieden Theoretiker wie auch Praktiker ihn deutlich von dem der «composición». ${ }^{3}$ In anderen europäischen Ländern, etwa in Frankreich, nannte man die Praxis «chanter sur le livre», «descant» in England, «sortisatio» in Deutschland und «contrappunto alla mente» in Italien und umfasste eine Gruppe von Sängern, die sich um das Pult versammelte: Ein Teil der Gruppe sang die Choralmelodie, wie sie in dem vor ihnen aufgeschlagenen Choralbuch notiert war, während einer der Sänger dafür zuständig war, eine zweite Melodielinie hinzuzufügen, woraus ein spontanes Duett anstelle des einstimmigen Gesanges entstand. Gelegentlich entschieden sich auch zwei, drei oder sogar mehr Sänger dazu, eine eigene, andere Melodie über den von der Gruppe getragenen Choral zu singen und ließen damit einen polyphonen Satz zu drei, vier oder mehr Stimmen entstehen. ${ }^{4}$

Einige Lehrmethoden und Traktate der Renaissance erwähnen dieses Verfahren und enthalten teilweise Passagen, in denen manche der Techniken beschrieben sind, die beim «Singen über dem Buch» Anwendung fanden. Die ausführlichste aus jener Zeit erhaltene Abhandlung stellt ein gegen Mitte des 16. Jahrhunderts geschriebener Traktat des portugiesischen Sängers und Komponisten Vicente Lusitano dar. Er ist fast ausschließlich dem contrapunto gewidmet und enthält über 200 Musikbeispiele, die helfen nachzuvollziehen, wie die Sänger improvisieren sollten. ${ }^{5}$

3 Zur Unterscheidung von contrapunto und composición in Spanien im 16. Jahrhundert siehe Giuseppe Fiorentino, »Con ayuda de nuestro señor: Teaching Improvised Counterpoint in Sixteenth-Century Spain«, in: New Perspectives on Early Music in Spain, hrsg. von Tess Knighton und Emilio Ros-Fábregas, Kassel 2015, S. 356-379.

4 Siehe zur Praxis des Cantare super Librum meine Monographie Limprovisation polyphonique à la Renaissance, Paris 2015 und die weiterfuihrende Literatur.

5 Für eine moderne Ausgabe des Traktates mitsamt transkribierter Musikbeispiele siehe Philippe Canguilhem (Hrsg.), Chanter sur le livre à la Renaissance. Les traités de contrepoint de Vicente Lusitano, Turnhout 2013. 
Das erste Kapitel lehrt, wie einem Choral eine weitere Stimme hinzuzufügen ist, und zwar zuerst im strengen >Note gegen Note<-Kontrapunkt und dann in freier Form. Sobald der Sänger die Grundlagen des freien Kontrapunkts beherrschte, unterrichtete man ihn im Singen in Begleitung: Das zweite Kapitel erläutert, wie zwei, drei und sogar vier Sänger gemeinsam über eine Choralmelodie improvisieren können, während das dritte und vierte Kapitel zum Einzelstudium und zu besonders komplexen, als abilidades bezeichneten Übungen zurückkehrt. Diese verschiedenen Fertigkeiten werden in zwei unterschiedliche Kategorien eingeteilt: Im dritten Kapitel erläutert Lusitano, wie ein Kanon unisono, mit dem Abstand einer Quarte, einer Quinte und sogar einer kleinen Sekunde »in Echtzeit« über dem Choral aufzubauen ist, und schließlich ist das letzte Kapitel der Improvisation mehrerer abilidades über mensurierte Musik gewidmet.

Es fällt auf, dass alle von Lusitano in dessen Traktat beschriebenen Übungen noch 70 Jahre später in Francisco Correas Abhandlung anzutreffen sind. Das zeigt eindeutig, dass der improvisierte Kontrapunkt zu den wesentlichen musikalischen Fähigkeiten gehörte, die ein kundiger Musiker in der Renaissance zu beherrschen hatte, weshalb auch ein beträchtlicher Teil der im 16. Jahrhundert erklungenen Musik nie zu Papier gebracht, sondern vielmehr von Sängern, die ex tempore «über dem Buch» sangen, realisiert worden sein dürfte.

Um deren Klanglichkeit zu rekonstruieren, bietet es sich an, den Anweisungen jener Traktate zu folgen, die sich, wie etwa Lusitanos Handschrift, dieser Praxis widmen. Im vorliegenden Aufsatz soll das Hauptaugenmerk auf einer bestimmten, nämlich der zweiten von Correa besprochenen Übung liegen, in der die Bewerber dazu aufgefordert sind, eine dritte, vierte und fünfte Stimme über einen bestehenden, mehrstimmigen Satz zu improvisieren.

Seinen ausführlichen Überblick über die verschiedenen, dieser Kategorie angehörigen Übungen beginnt Correa mit der Erläuterung, wie eine dritte Stimme ex tempore zu einem bestehenden Duett kreiert werden kann:

»- Die dritte Stimme kann auf zwei verschiedene Arten zu einem duo gesungen werden: darüber oder darunter. - Wird die untere Stimme des duo um eine Oktave höher gesungen, soll die dritte Stimme als Bariton (bajete) improvisiert werden, der beide Stimmen abdeckt. ${ }^{6}$

Von den drei Übungen, die Correa vorschlägt, erscheint diese dritte heute durchaus ungewöhnlich. Sie könnte auf die damalige Situation zurückzuführen sein,

6 »Tercera voz sobre un duo como esta puntado en dos maneras: una es por encima y otra por abajo. Item, tercera vos, trocando las vozes, que es cantando la baja octava arriba de como esta puntada, echarle un bajete que las cubra«. Preciado, »Un documento nuevo« (wie Anm. 1), S. 47. 
in der einigen Kapellmeistern Männerstimmen im Chor fehlten und sie daher auf Kinderstimmen angewiesen waren, um mehrstimmig singen zu können. Die Transposition der unteren Stimme eines Duetts um eine Oktave nach oben und das Hinzufügen einer Bassstimme hätte ein Terzett entstehen lassen, dessen beide obere Stimmen von Kindern gesungen wurden.

Die Tatsache, dass diese Übung in der darauffolgenden Aufgabenserie wiederholt und dort als »wesentlichst « (esencialisima) beschrieben wird, scheint diese These zu unterstïtzen:

»Die vierte Stimme, das ist die wesentlichste Fähigkeit: Einem tercio soll eine vierte, fehlende Stimme hinzugefügt werden. Item, wenn die tiefste Stimme um eine Oktave erhöht ist: Das ist die wichtigste Übung, die gemeistert werden muss. - Item, bei einem cuatro, verdecke den Bass und improvisiere ihn. $\ll^{7}$

Wenn auch Correa die Improvisation einer vierten Stimme zu einem bestehenden Terzett als die wesentlichste Fähigkeit bezeichnet, schließt der spanische Organist nicht an dieser Stelle, sondern fordert eine letzte »notwendige« Reihe von Übungen:

$»$ Die fünfte Stimme. Singe eine fünfte Stimme zu einem notierten cuatro. Item, wenn der Bass um eine Oktave erhöht ist. Diese sind zwei beeindruckende Fähigkeiten, die nur wenige beherrschen, und dann wirkt es fast, als brüsteten sie sich damit. ${ }^{8}$

Und Correa beendet dieses Kapitel: «Es können noch weitere Übungen verlangt werden, doch dienen sie vielmehr der Prahlerei, wie etwa das Improvisieren einer sechsten, siebten oder sogar einer achten Stimme: Korrekt ausgeführt, stellt dies eine erstaunliche Fähigkeit dar. Dennoch sind diese Fertigkeiten nicht notwendig.» ${ }^{9}$

$\mathrm{Zu}$ einem bestehenden polyphonen Satz eine weitere Stimme hinzuzufügen, stellte in der Renaissance ein gängiges, kontrapunktisches Verfahren dar, das auch durch notierte Musikquellen gut belegt ist. Die meisten Fälle betreffen dreistim-

$7 \gg$ Cuarta voz, y esta es la habilidad esencialisima. Item, se ha de echar cuarta voz sobre un tercio, como el esta puntado, echandole la voz que le falta. Item cuarta voz, cantando octava arriba la voz mas baja del tercio, y esta es la mas necesaria y la que se ha de hacer mejor. Item, a un cuatro, tapanle el bajo y que lo eche«.

8 »Quinta voz. Echar quinta voz sobre un cuatro como el esta puntado. Item, que el bajo se diga octaba arriba, y de este modo se le eche quinta boz. Estas dos son grandes habilidades, y pocos las hacen bien, y casi es ostentativa

9 »Otras hay de mayor ostentacion, que es echar sexta y septima y aun octava voz, y si hace limpiamente, es cosa prodigiosa. No son necesarias«. 
mige Stücke, die in den Quellen gelegentlich mit einer optionalen vierten Stimme erscheinen, die mit si placet gekennzeichnet ist, einem Hinweis darauf, dass jene vierte Stimme zu einem späteren Zeitpunkt, nachdem die ursprünglich dreistimmige Komposition fertiggestellt war, entstanden ist. Von wenigen Ausnahmen abgesehen, sind für diese hinzugekommenen Stimmen keinerlei Zuschreibungen erhalten, ein Umstand, auf den am Ende dieses Aufsatzes noch einzugehen sein wird.

Diese für das 15. Jahrhundert umfassend belegte Praxis findet die ganze Renaissance hindurch Verwendung und ist heute als »si placet-Komponieren« bekannt. Der Definition von Stephanie Schlagel zufolge umfasst diese besondere Form der kompositorischen Bearbeitung (»special category of compositional reworking «) die Erschaffung neuer Stimmen, die zu einer darüber hinaus unverändert bleibenden Komposition hinzutreten (»the creation of newly composed voices that are added to an original composition, which otherwise remains unaltered «). ${ }^{10}$ Bislang wurde die si placet-Tradition als Kompositionspraxis und ihre vielfach erhaltenen Belege als ihr genuines »Repertoire « verstanden. ${ }^{11}$ Francisco Correas Abhandlung bezeugt jedoch, dass die besten Musiker die hinzukommende Stimme unmittelbar singen, das heißt improvisieren sollten. In der Tat sprechen die zeitgenössischen Theoretiker diesbezüglich nie vom Komponieren, sondern von einer spezifisch mündlichen Improvisationsmethode.

Wie war es möglich, spontan eine neue Stimme zu einem mehrstimmigen Satz zu singen? Welchen Schwierigkeiten stand man dabei gegenüber? In welchem Maße können die erhaltenen Beispiele von si placet-Stimmen helfen, den Improvisationsstil ihrer Autoren zu rekonstruieren? Diese Fragen sind, zumal im Rahmen eines kurzen Aufsatzes, nicht leicht zu beantworten. In den folgenden Abschnitten werden dazu die theoretischen Schriften, die sich mit dieser Praxis befassen, konsultiert, da hieran ein erster Schritt auf dem Weg zum Verständnis dessen festzumachen ist, was ich »si placet-Singen« nennen will.

Das si placet-Singen wird von zwei Theoretikern der Renaissance im Detail behandelt: von dem bereits genannten Vicente Lusitano sowie von Gioseffo Zarlino. Lusitano erläutert zu Beginn anhand des Et resurrexit aus dem Credo von Nicolas Gomberts Missa Philomena als Beispiel für die Regeln und Anweisungen, wie einem Duett eine dritte Stimme hinzuzufügen sei. Zu einem Duett eine Mittelstimme zu erzeugen, ist Lusitano zufolge «schwierig, wird bei richtiger Ausführung aber geschätzt». Damit dies gelinge, erklärt er, solle man sich auf den Bass konzentrieren, zugleich die andere Stimme prüfen, dabei Sexten über dem Bass vermeiden, wohl aber Oktaven, Quinten und Terzen abwechseln. Dies

10 Stephanie P. Schlagel, Si placet Parts for Motets by Josquin and His Contemporaries, Madison 2006, S. ix.

11 Siehe die oben angeführte Ausgabe von Schlagel sowie Stephen Self, The si placet repertoire of 1480-1530, Madison 1996. 
könne nur mit einem schnellen und guten Gehör sowie Kompositionserfahrung funktionieren. ${ }^{12}$

Noch etwas schwieriger sei es hingegen, wenn die Stimme unter dem Satz ergänzt wird. In seinen eigenen Worten: «Die zweite Möglichkeit besteht darin, eine tiefe zu zwei hohen Stimmen zu singen, was über zwei Stimmen schwierig und sehr lobenswert ist. Wenn dies aber über drei Stimmen gelingt, erreichen wir den Höhepunkt der Fähigkeiten, da es in der Musikpraxis keine herausragendere Fertigkeit gibt als diese. ${ }^{13}$ Lusitano fährt mit einigen technischen Ratschlägen bezüglich der Ergänzung einer tiefen Stimme zu einem Duett fort: Der Sänger solle bei dieser Übung den Sopran oder die höchste Stimme anvisieren und, sofern es ihm beliebt, hauptsächlich parallele Dezimen darunter singen. Noch besser sei es aber, wenn er durch Aufgreifen melodischer Motive auch die übrigen Stimmen imitieren könne. ${ }^{14}$

Wie noch zu sehen sein wird, sind diese grundlegenden Empfehlungen - vornehmlich auf den Bass zu achten, wenn eine Mittelstimme ergänzt wird und auf den Sopran zu achten, wenn eine tiefe Stimme hinzutritt - äußerst informativ für uns, stellen sie nach meiner Kenntnis die einzige erhaltene, technische Bemerkung dazu dar, wie eine dritte Stimme zu einem Duett hinzugefügt werden kann. ${ }^{15}$

Vor diesem Hintergrund ist seine Besprechung des nächsten Schrittes noch aufschlussreicher. Zu der Beschreibung, wie eine vierte Stimme einem Terzett hinzuzufuigen sei, zieht Lusitano ein anderes Musikbeispiel heran, das dreistimmige Crucifixus aus Gomberts Missa Philomena. Die ersten Erläuterungen befassen

12 Canguilhem (Hrsg.), Chanter sur le livre à la Renaissance (wie Anm. 5), S. 283: »echando sobre la boz mas baxa con oyo en la segunda y sus terminos y guar- dandose que no eche muchas sextas, o quasi ninguna, mas continuando las octavas, quintas, terçeras y sus conpuestas, haran dulçe conçento. La qual abilidad proçede del mucho uso de la conpostura y viveza de oydo, con las quales se junta la grande destreza sobre una boz.«

13 Ebda.: »La segunda manera es echar un contrabaxo a bozes altas, la qual hecha sobre dos bozes es mucho y muy de loar. Mas si sobre tres se haze bien, es el fin de todas las abilidades, y no ay major en la musica pratica.«

14 Ebda.: »Para lo qual nota que la boz que se echa sienpre deve mirar al tiple o a la boz mas alta, y usar de muchas dezenas con ella, excepto si alguna otra boz se subiere en alto del tiple, ca entonçes a la mas alta se mirara como en la primera manera a la mas baxa. Mas es de notar que si se echa sobre dos la boz baxa puede sienpre hazer dezenas, exçepto si las dos bozes hazen muchas sextas, ca entonçes de otras species deve usar, y esto por que haziendo en tal parte dezenas seran con la segunda boz quintas. « Und S. 286: »Si la boz que es echada de inproviso pudiere buenamente ymitar a las otras, develo de hazer; y si no, deve tomar algun paso, el qual deve replicar algunas vezes, segun que se mostro en las boxes arriba puestas sobre el duo, por que esta es la meior manera para cantar con graçia de inproviso.«

15 Die beiden von Lusitano angebrachten Beispiele (die dritten Stimmen zu Et resurrexit und Crucifixus) sind entweder in originaler Schlüsselung in Canguilhem (Hrsg.), Chanter sur le livre à la Renaissance (wie Anm. 5), S. 283-289 verfügbar oder stehen in moderner Schlüsselung auf der Webseite RICERCAR des Centre d'Études Supérieures de la Renaissance an der Universität von Tours zum Download bereit: http://josquin.cesr.univ-tours.fr/lusitano/collections/show/5 (Beispiele 72, 73 and 74). 
sich damit, wie eine Mittelstimme zu dem Terzett zu improvisieren sei. Zudem gibt Lusitano verschiedene Ratschläge zur Beschaffenheit der zusätzlichen Stimme, handele es sich nun um einen Sopran, Alt oder Tenor. Schließlich kommt er darauf zurück, wie eine Bassstimme zu einem präexistenten Terzett gesungen werden könne, und damit zu jener Technik, die Francisco Correa noch fast ein Jahrhundert später als esencialisima für einen Kapellmeister und als das höchste Geschick in der ausübenden Musik erachtete. ${ }^{16}$ Lusitanos Leser wird allerdings enttäuscht, da der Verfasser zugeben muss, dass aufgrund der extremen Schwierigkeit dieser Aufgabe keine technische Anweisungen dazu geben werden können:

»Wenn aber die vierte Stimme als Bass gesungen wird, kann kein weiterer Ratschlag gegeben werden, als auf die übrigen drei Stimmen zu achten und das Gehör anzustrengen, um deren Motive zu imitieren und auf die Kadenzen zu hören, da sich die Kadenzbildung mit einer ergänzenden Bassstimme zu drei ausgeführten Stimmen schwierig gestaltet. Daher zeige ich hieran anschließend, wie man eine hohe und eine tiefe Stimme zu einem Terzett hinzufügt. « ${ }^{17}$

Die Schwierigkeit dieser Übung liegt darin begründet, dass in einem notierten Terzett alle für den Bass charakteristischen Melodie- und Kadenzwendungen durch die aufgeschriebene, tiefe Stimme übernommen werden und es sich aus diesem Grund schwierig gestaltet, eine passende Melodielinie für den zusätzlichen Bass zu finden. Das von Lusitano dafür ausgewählte Beispiel (Beispiel 1) zeigt, wie er mit verschiedenen solcher Fälle (siehe Hervorhebungen) umgeht: Die erste, phrygische Kadenz auf $A$ wird durch Einfügen eines $G$ im Bass zur plagalen Kadenz in $D$ umgewandelt; in der zweiten - perfekten - Kadenz auf $A$ wird die dritte Stimme verdoppelt und »verschwindet« dann, um ein Unisono oder parallele Oktaven zu vermeiden; die darauffolgende, perfekte Kadenz auf $D$ wird, da sie ursprünglich für zwei Stimmen notiert ist, durch die Bassstimme vervollständigt; die Kadenz des Tenors auf $F$ im letzten System wird zur plagalen Kadenz auf $D$ umgeformt; und schließlich wird die letzte perfekte Kadenz zu einer plagalen Kadenz umfunktioniert, indem eine Bassbewegung von $B$ zu $G$ ergänzt wird, die den $D$-Schlussakord zum vorletzten Akkord über $G$ werden lässt, . Durch diese Ergänzung zögert Lusitano die urspüngliche Kadenz um eine Semibrevis hinaus.

16 Siehe Anm. 13.

17 »Mas, si la quarta parte se echa en baxo, aqui no ay otro aviso que dar, sino que la quarta parte deve ser avisado de guardar a todas las tres y aver grande oydo para los pasos que pueden responder y a las clausulas, por que son muy dificultosas las clausulas del baxo echado sobre tres partes conçertadas, y por esto brevemente se pondra el modo como se echara una quarta boz en alto y en baxo.« 

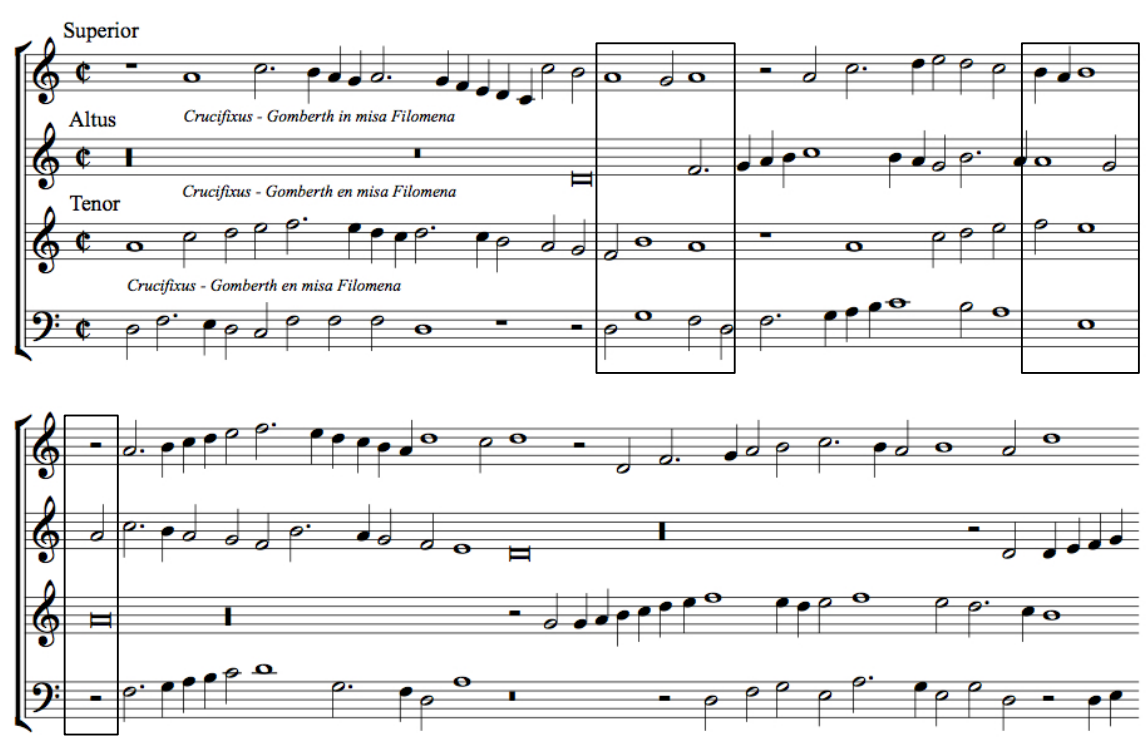

Notenbeispiel 1. Nicolas Gombert, Missa Philomena, »Crucifixus«, mit hinzugefügter vierter Stimme im Bass von Vicente Lusitano (Kadenzen hervorgehoben).

Trotz des hohen Wertes, der Lusitanos Anleitungen und Musikbeispiele auszeichnet, ist er nicht der einzige, der sich dieses Gegenstandes im 16. Jahrhundert annimmt, denn weniger als zehn Jahre später widmet Gioseffo Zarlino der si placet-Praktik im dritten, mit L'arte del contrapunto betitelten Teil seiner Istitutioni harmoniche ein ganzes Kapitel. Angesichts von Zarlinos Reputation und der Rezeption seines theoretischen Werks in den Jahrzehnten zuvor überrascht seine Auseinandersetzung mit Improvisationstechniken vielleicht zunächst, wurde seine Kontrapunktlehre doch in enger Verbindung zur Verschriftlichung gesehen. Der Titel des untersuchten Kapitels, "Quel che si dè osservare, quando si volesse fare una terza parte alla sproveduta sopra due altre proposte « (Was zu beachten ist, wenn man eine dritte Stimme ohne Vorbereitung zu zwei bestehenden Stimmen singen will), lässt allerdings keinen Zweifel an dem Improvisationscharakter dieser Praxis. ${ }^{18}$

In der Tat betont Zarlino die Probleme, die sich durch eben diese Spontaneität ergeben. Seiner Meinung nach müssten jene, die diese Übung meistern wollen,

18 Gioseffo Zarlino, Le istitutioni harmoniche, Venedig 1558, Kapitel 64: Quel che si dè osservare, quando si volesse fare una terza parte alla sproveduta sopra due altre proposte. Englische Übersetzung: Gioseffo Zarlino, The Art of Counterpoint, hrsg. von Guy A. Marco and Claude V. Palisca, New Haven und London 1968. 
ihre zusätzliche Stimme durch gründliche Betrachtung des bestehenden Duetts sorgfältig im Voraus vorbereiten:

»Er sollte die Passagen und Modulationen der beiden vorgegebenen Stimmen solange studieren, bis er vollends begreift, wie der Kontrapunkt aufgebaut ist. Erst dann ist er dazu in der Lage, eine Stimme seiner Wahl fehlerfrei hinzuzufügen. ${ }^{19}$

In den nachfolgenden Erläuterungen entfernt sich Zarlino in zwei wesentlichen Punkten von Lusitano. Zum einen verzichtet Zarlino auf technische Ratschläge, etwa zu der Abfolge von Intervallen oder den melodischen Stil, den die hinzukommende Stimme übernehmen sollte, und verfolgt stattdessen eine allgemeine Diskussion über die Methode, mithilfe derer es gelingen kann, ein Duett um eine dritte Stimme zu ergänzen. Auch diese Methode unterscheidet sich deutlich von Lusitanos Vorschlägen. Zarlino zufolge »genügt (entgegen der Auffassung vieler Ungelehrter) die Betrachtung einer Stimme allein nicht, um den Kontrapunkt zu bestimmen, der mit der dritten Stimme hinzutreten soll «, ${ }^{20}$ wohingegen Lusitano seinem Leser dazu rät, entweder die tiefere oder die höhere Stimme anzusehen.

Zum anderen differieren Zarlinos und Lusitanos Auffassungen bezüglich eines von Lusitano empfohlenen Verfahrens, nämlich der Möglichkeit, parallele Dezimen zu einer der beiden Duettstimmen zu singen. Zarlino steht dieser Praxis höchst kritisch gegenüber und bezeichnet solche Musiker, die Dezimparallelen zu einer Stimme des polyhphonen Satzes ausführten und damit die Zuhörer mit einer Fähigkeit zu beeindrucken beabsichtigten, über die sie gar nicht verfügten, als anmaßend und arrogant. »Sie versuchen damit diejenigen, die so dumm sind wie sie selbst, glauben zu machen, sie könnten Wunder wirken. $\ll^{21}$

19 The Art of Counterpoint, S. 221; Istitutioni harmoniche, S. 258: »Debbe con diligenza por mente alli passaggi, et alle modulationi che fanno insieme le due parti proposte, acciò possa comprendere in che maniera il loro contrapunto sia ordinato, et possa dipoi aggiungere senza alcuno errore quella parte, che lui vuole.«

20 The Art of Counterpoint, S. 221 f.; Le istitutioni harmoniche, S. 258: »perché non è sufficiente (come si avisano molti che non sanno) una parte sola a mostrare il contrapunto che si hà da aggiungere nella terza parte.

21 The Art of Counterpoint, S. 221; Le istitutioni harmoniche, S. 258: »ho udito alcuni, non dirò sciocchi, ma presuntuosi affatto et arroganti, che per dare ad intendere che sono in ciò molto valorosi et sufficienti [...] danno ad intendere alli sciocchi, come sono loro, e che non intendeno più oltra, che fanno miracoli.« 

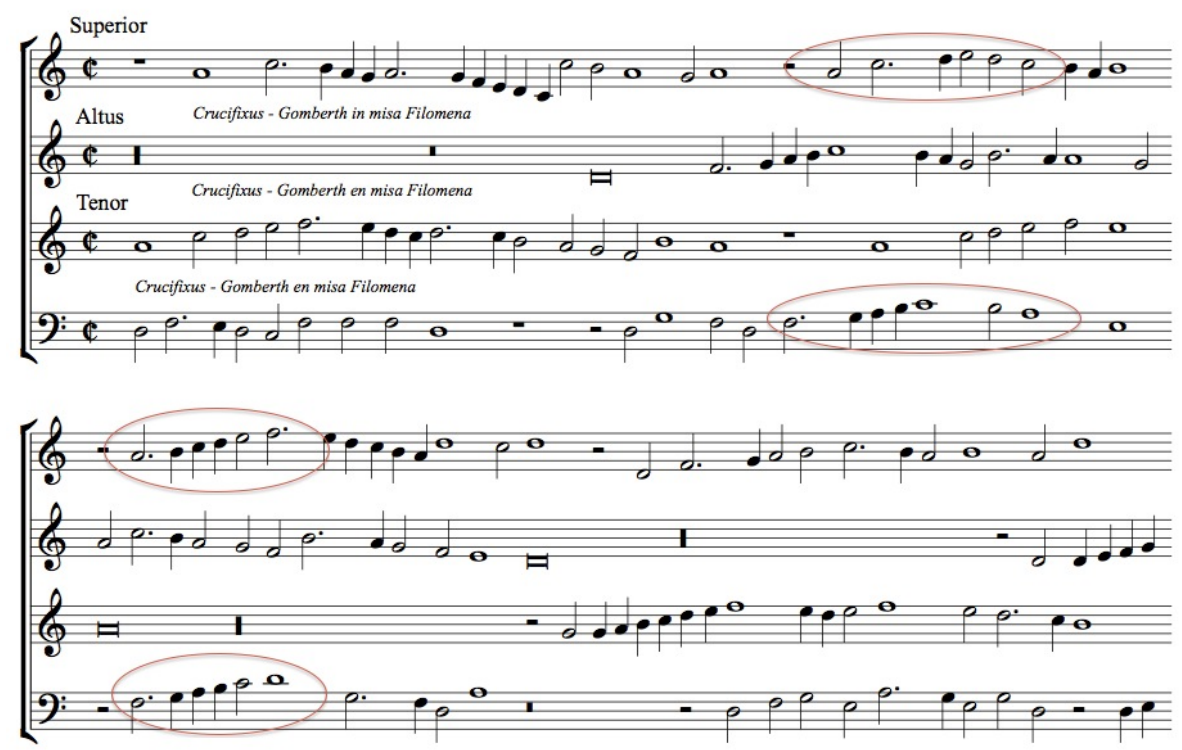

Notenbeispiel 2. Nicolas Gombert, Missa Philomena, „Crucifixus«, mit hinzugefügter vierter Stimme im Bass von Vicente Lusitano (Dezimen hervorgehoben).

Doch zurück zu Lusitanos Musikbeispiel (Notenbeispiel 2): Obwohl hier parallel unter dem Sopran geführte Dezimen präsent sind, kann sein si placet-Bass bei Weitem nicht unter diesem Gesichtspunkt allein betrachtet werden. Daran wird ersichtlich, dass Lusitano sich nicht darauf hätte beschränken können, zur Erzeugung der vierten Stimme nur den Sopran von Gomberts Crucifixus zu berücksichtigen. Das si placet-Singen war deshalb eine derart schwierige Aufgabe, weil es die auf der Chorbuchseite verteilten Stimmen nahezu unmöglich machten, deren kontrapunktische Zusammenhänge untereinander zu erfassen. Zarlinos Musikbeispiele (Abbildung 1) zeigen in ihrer ursprünglichen Disposition die beiden Stimmen von Josquins Per illud Ave prolatum, eine neben der anderen, auf der Seite getrennt voneinander. Er brachte sie folglich nicht einmal in die Form eines »quasi-score« oder »pseudo-score«, wie Jessie Ann Owens die Anordnung bezeichnet, in der die Stimmen in einer Reihe untereinander, jedoch ohne Taktstriche und vertikale Angleichung positioniert sind. ${ }^{22}$

22 Siehe Jessie Ann Owens, Composers at Work, New York und Oxford 1997, S. 34-38. 
Partè.

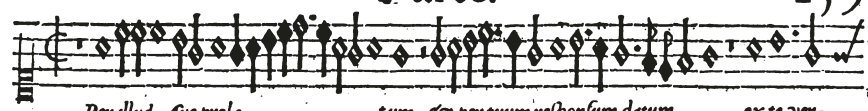

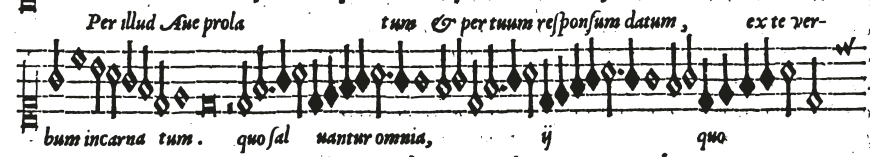

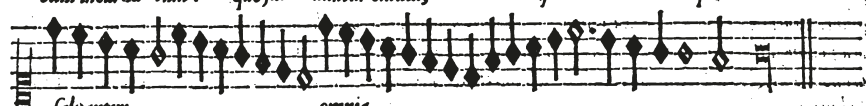

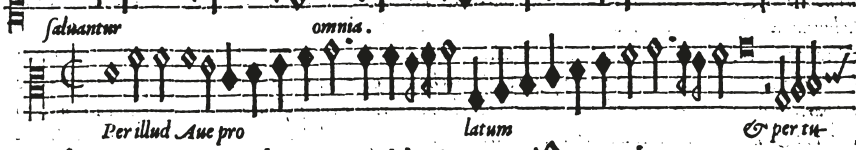

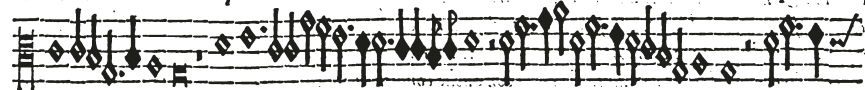
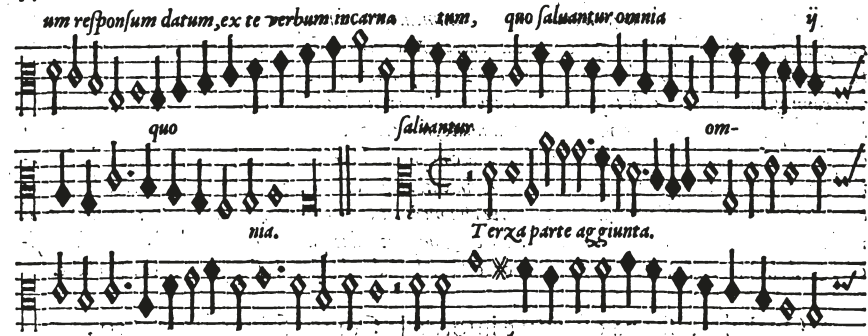

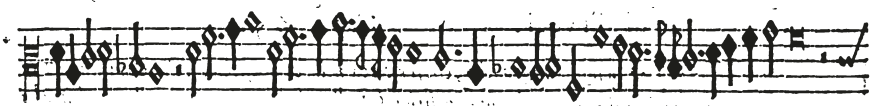
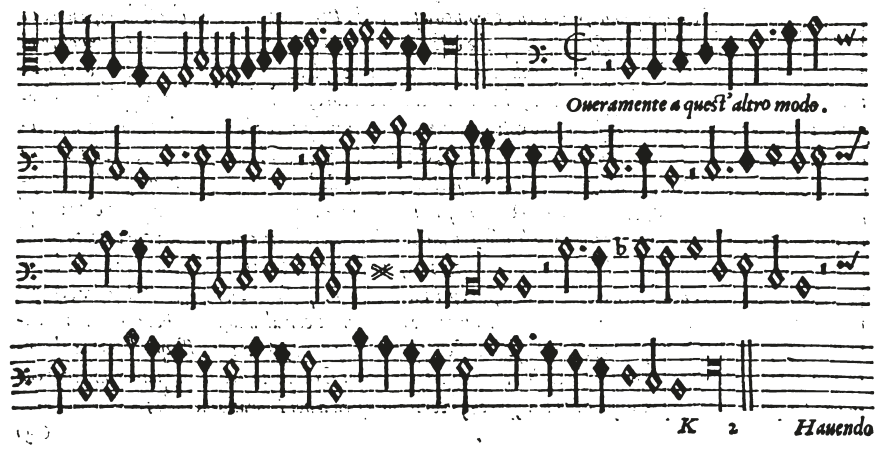

Abbildung 1. Gioseffo Zarlino, Le istitutioni harmoniche, Venedig 1558, S. 259: Josquin duo Per illud Ave prolatum mit zwei verschiedenen von Zarlino hinzugefügten dritten Stimmen. 
Das erklärt auch, warum die Improvisation einer dritten Stimme als Fertigkeit erfahrener Musiker galt und auch für die besten unter ihnen immer wieder eine Herausforderung darstellte. Davon zeugt eine von Lodovico Zacconi in der zweiten Ausgabe seiner 1622 veröffentlichten Prattica di musica übermittelte Anekdote, in der Zacconi in einem Abschnitt des Traktates davon berichtet, dass Zarlinos hochverehrter Lehrer, Adrian Willaert, folgende Übung mit seinen Schülern praktizierte:

»Ich erinnere mich daran, wie mir Gioseffo Zarlino eines Tages davon berichtete, dass sein Meister Adriano, wenn er die dritte Stimme über ein Duett improvisiert hatte, es häufig noch ein zweites Mal tat und sagte: >Diesmal habe ich es gut gemacht, nicht aber beim ersten Mak. Damit wollte er demonstrieren, dass man bei der Improvisation nicht dasselbe tut, wie wenn man einen Stift in der Hand hätte, weil man nicht voraussehen und überlegen könne, was man singt. $\ll^{23}$

Sicherlich sah sich Zarlino aufgrund dieser Schwierigkeit dazu angeregt, das si placet-Singen auf die dritte und keine weitere Stimme zu begrenzen, und tatsächlich präsentiert er sein Musikbeispiel, Josquins Duett, mit zwei unterschiedlichen dritten Stimmen, einer Stimme in der Mitte und einer unterhalb des Duetts. ${ }^{24}$ Verschmähte er 1558 noch jene, die willens waren, eine zusätzliche Stimme zu einem mehr als zweistimmigen Satz zu singen und beschrieb sie missachtend als arrogant und anmaßend, relativierte er seine Aussage in einem letzten Satz, den er am Ende des Kapitels der 31 Jahre später im Jahre 1589 erschienenen letzten Ausgabe seines Traktates hinzufügte:

»Was ich über das Hinzufügen einer Stimme zu zweien gesagt habe, ist ebenso auf den Fall von drei oder gar vier Stimmen zu übertragen. Es ist zwar eine anspruchsvolle, doch keine unlösbare Aufgabe und es stimmt, dass die Schwierigkeit für denjenigen, der eine Stimme hinzufügen möchte, zunimmt, je größer die Stimmenzahl des vorliegenden Satzes ist. Drei Dinge sind [dafür] notwendig: ein gutes Gedächtnis, konstantes Üben und ein gutes Gehör, um die übrigen Stimmen des Satzes, zu dem die Stimme

23 Lodovico Zacconi, Prattica di musica seconda parte, Venedig 1622, S. 153 f.: »Ed insimil proposito raccontò che il signor Adriano suo maestro nel far ch'egli facea una terza parte sopra un duo alla mente, fatto che ve l'havea una volta, bene spesso ve lo rifacea di nuovo e poi dicea: adesso io l'ho fatta bene, e non la prima voltar. Volendo in questo mostrare ch'all'improviso l'huom alle volte vi fa cose che non si farebbono quando che si havesse la penna in mano, o che l'huom meglio le potesse antevedere e considerare.«

24 Siehe Schlagel, Si Placet Parts (wie Anm. 10), S. 152-154 für eine moderne Ausgabe von Zarlinos zu Josquins Duett hinzugefügten Stimmen. 
hinzutreten soll, schnell erfassen zu können. Ohne diese Dinge ist nichts oder nur wenig auszurichten. $\ll^{25}$

Stand Zarlino 1558 also denjenigen, die versuchten, über ein Terzett oder Quartett zu improvisieren, noch kritisch gegenüber, war dies gegen Ende seines Lebens nicht mehr der Fall. Doch womit ist diese Entwicklung zu erklären? Möglicherweise wurde der Sinneswandel durch die Ausbreitung dieser Praxis ausgelöst, die das si placet-Singen in der zweiten Hälfte des 16. Jahrhunderts erlebte und dazu fuihrte, dass die besten Musiker ihre Techniken weiter verbesserten. Die eigene Anschauung solcher Musiker könnte Zarlino davon überzeugt haben, diese Praxis für erwähnenswert zu befinden. Diese historische Argumentation steht wiederum in Widerspruch zu den Schriftquellen der si placet-Stimmen, die nämlich nach 1530 aus Handschriften und Drucken verschwinden. Zeichnet man eine Linie zwischen Zarlinos Meinungsänderung und dem, was Francisco Correa zuletzt 1637 niederschrieb, scheint es, dass die Praxis, eine Stimme über schriftlich fixierte, mehrstimmige Musik zu improvisieren, sich am Ende des 16. Jahrhunderts großer Beliebtheit erfreute.

Selbstverständlich müssen auf diese erste Annäherung an das si placet-Singen und die damit in Verbindung stehenden Fragen weiterführende Nachforschungen folgen, von denen eines der anzugehenden Probleme die performative Seite dieses Verfahrens betreffen sollte. In der Tat dürfen die Musikbeispiele von Lusitano oder auch jene von Zarlino nicht als Kompositionen, sondern müssen als Improvisationen angesehen werden. Dieser Umstand verändert unsere Sicht auf die dabei entstandene Mehrstimmigkeit: Während der Ausführung wird man nämlich nicht auf den Satz als Ganzen, sondern viel eher auf die eine hinzukommende Stimme achten und darauf, wie sie sich in das Gesamtgefüge integriert oder aus ihm heraussticht. Aus diesem Grund ist das si placet-Singen weniger als Hommage an das ursprüngliche Werk denn als Vorwand für die Demonstration der eigenen kontrapunktischen Fähigkeiten zu verstehen.

Betrachtet man die große Zahl von schriftlich tradierten si placet-Stimmen nicht als Kompositionen im engeren Sinne, sondern als Zeugnis einer Aufführungspraxis, wird man auch erkennen, dass sich in den überlieferten stilistischen Unterschieden im Grunde Fertigkeiten in der Ausführung spiegeln. Dass sie

25 Dieser Zusatz erscheint am Ende des gleichen Kapitels in Gioseffo Zarlino, Tutte le opere, Venedig 1589, S. 336: »Et quello chò detto dell raggiungere una parte a due, si può anco intendere, quando se ne volesse aggiungere una a tre, et anco a quattro. Percioché non è cosa impossibile, se ben è difficile. È ben vero, che quanto più sarà il numero delle parti, tanto più difficultà apporterà a colui, che vorrà aggiungere cotale parte. Ma bisogna sopra il tutto tre cose: buona memoria, e lunga essercitatione, e buon occhio per potere raccogliere con prestezza le parti della cantilena, sopra le quali sshavrà da aggiungere cotal parte. Senza le quali cose, nulla o poco almeno si farebbe.« 


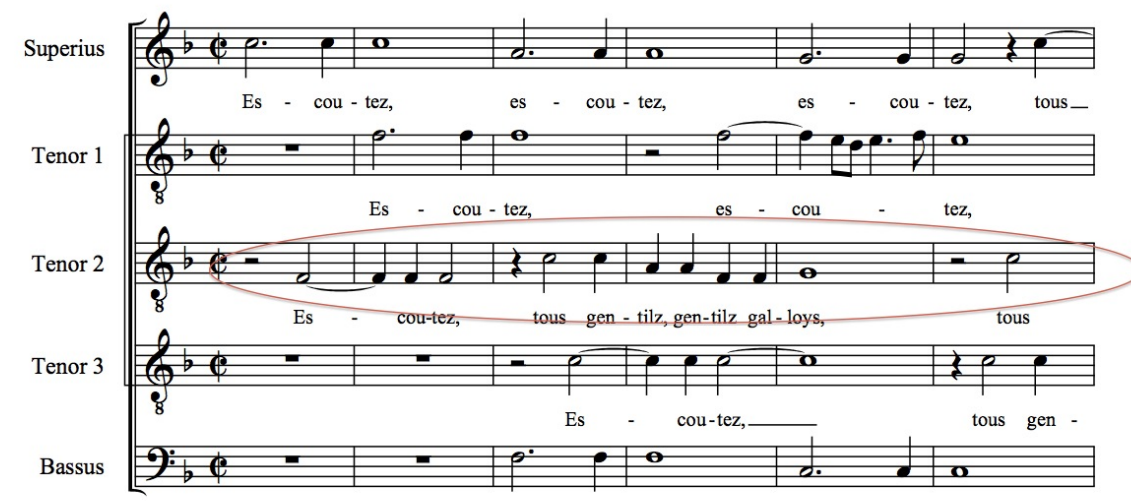

Es - cou - tez, es - cou - tez,

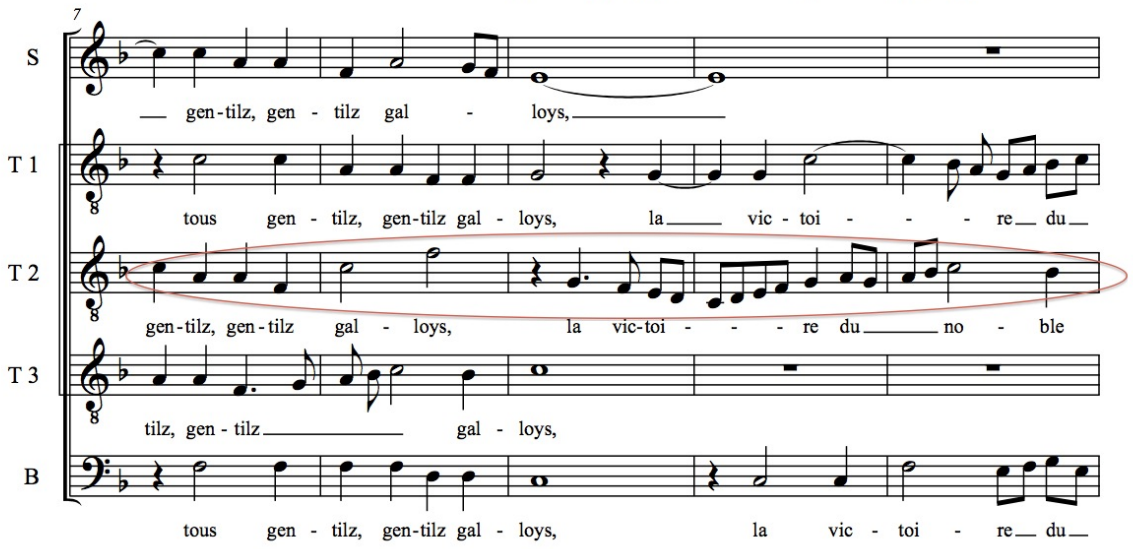

Notenbeispiel 3. Clément Janequin, La bataille, mit von Philippe Verdelot hinzugefügter fünfter Stimme.

weniger Kompositionen als vielmehr Relikte des Improvisationsstils eines Sängers darstellen, könnte zum einen erklären, weshalb das Repertoire zu größten Teilen ohne Zuschreibung überliefert ist. Zum anderen wird auch die stilistische Vielfalt verständlich, die die in den Schriftquellen erhaltenen si placet-Stimmen kennzeichnet: Einige von ihnen versuchen, durch Imitation der melodischen Motive Bestandteil des polyphonen Satzes zu werden und diesen auszubauen, wie etwa in Bidons sechster Stimme zu Josquins Miserere oder auch der fünften Stimme von Verdelot zu Janequins La bataille (Notenbeispiel 3) zu sehen ist. ${ }^{26}$

26 Bidons sechste Stimme zu Josquins Miserere ist in Schlagel, Si Placet Parts (wie Anm. 10), S. 22 $62 \mathrm{zu}$ finden. Zu Bidon und mehrstimmiger Improvisation siehe Canguilhem, Limprovisation polyphonique (wie Anm. 4), S. 209-212. 
In anderen Fällen erwecken die hinzugefügten Stimmen hingegen den Anschein, als seien sie dazu bestimmt gewesen, die Virtuosität des Sängers hervorzuheben, wie der über zwei Oktaven reichende Ambitus in Huc me sidereo oder die Konstruktion eines Minimenkanons, der etwa in der bekannten, Josquin zugeschriebenen Version von De tous bien plaine anzutreffen ist, suggerieren (Notenbeispiel 4$).{ }^{27}$

In beiden Fällen gewähren die Beispiele von notierten si placet-Stimmen einen faszinierenden Einblick in eine Fertigkeit, die die besten Musiker des 15. bis 17. Jahrhunderts besaßen. In Verbindung mit den Traktaten und anderen Belegen, wie etwa Correas Parecer, kann die Betrachtung dieser Aufzeichnungen einen Schlüssel zum Verständnis einer der bemerkenswertesten Praktiken polyphonen Improvisierens in der Renaissance bieten.

27 Huc me sidereo, gelegentlich Josquin zugeschrieben, ist mit der anonymen sechsten Stimme ediert in Schlagel, Si Placet Parts (wie Anm. 10), S. 3-21. Zu Kanones als gebräuchliche Improvisationstechniken siehe Canguilhem, Limprovisation polyphonique (wie Anm. 4), S. 217-220 sowie die weiterführende Literatur. 

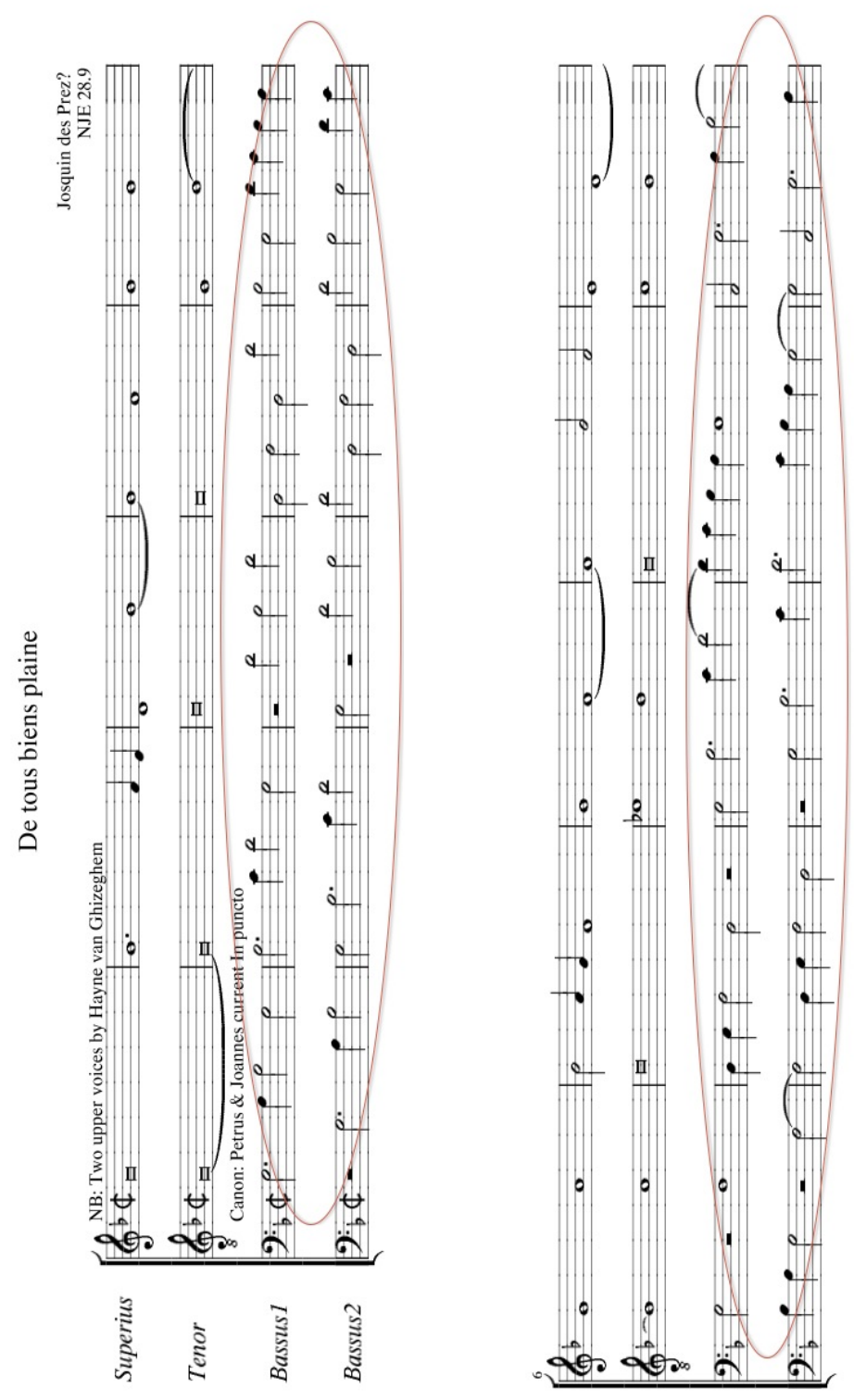

Notenbeispiel 4. Hayne van Ghizeghem, De tous biens plaine mit hinzugefügtem Kanon von Josquin Desprez. 